\title{
Microwave-assisted synthesis of ethyl 7-chloro-4-oxo-1,4-dihydro-1,8- naphthyridine-3-carboxylate by the Grohe-Heitzer reaction
}

\author{
Socorro Leyva-Ramos, ${ }^{* 1}$ Elisa Leyva, ${ }^{1}$ Jaime Cardoso-Ortiz ${ }^{2}$ and Hiram Hernández-López ${ }^{2}$ \\ 1 Facultad de Ciencias Químicas, Universidad Autónoma de San Luis Potosí, 78210, San Luis Potosí, SLP, México. \\ Tel: 524448262440 Ex 6476. e-mail: sleyva@uaslp.mx \\ 2 Unidad Académica de Ciencias Químicas, Universidad Autónoma de Zacatecas, 98160, Zacatecas, Zac. México.
}

Received September $2^{\text {nd }}, 2016$; Accepted January $17^{\text {th }}, 2017$.

\begin{abstract}
A simple and efficient two-step method was implemented for the synthesis of ethyl 7-chloro-4-oxo-1,4-dihydro-1,8-naphthyridine-3-carboxylate. Comparing with the reported Grohe-Heitzer reaction, the time and the reaction steps have been reduced using microwave irradiation. This compound is an important intermediate for the preparation of naphthyridone derivatives which have received significant attention due to their broad spectrum of biological activity. Keywords: Grohe-Heitzer reaction; 1,8-naphtyridone; microwave irradiation; tautomeric equilibrium.
\end{abstract}

\section{Introduction}

The 1,8-naphthyridone derivatives have received significant attention due to their exceptionally broad spectrum of biological activities. The first 1,8-naphthyridone group was nalidixic acid used as a synthetic quinolone antibiotic $[1,2]$. However, this quinolone showed activity only against Gram-negative microorganisms. Subsequent structural modifications of this quinolone led to a wide range of biological properties established them as potent scaffolds in therapeutic and medicinal research. The broad spectrum of activities includes antibacterial $[3,4]$, antitumor [5-9], antiviral [10, 11] and some antimycobacterial activities $[12,13]$ as well as anti-inflammatory activity [14-17]. The structural features of the more potent, newer generation quinolones such as tosufloxacin, trovafloxacin, enoxacin and gemifloxacin (Figure 1) include C-6 fluorine and a C-7 nitrogen-containing heterocycle including piperazine or pyrrolidine into the 1,8-naphthyridone group $[3,4,16]$.

In spite of a significant number of fluoroquinolones being approved for treatment of bacterial infections [18]; there is still need for developing new derivatives that could overcome the emerging problem represented by bacterial chemoresistance. Furthermore, some antibacterial quinolones have important significant side effects. For example, trovafloxacin was recently removed due to its liver toxicity [19].

Numerous synthetic methods have been reported for the preparation of quinolones under conventional conditions; however, these methods describe long reaction times, low yields and elaborated work-up procedures. In this paper, we describe a fast microwave-assisted synthesis for the preparation of ethyl
Resumen. Se implementó un método simple y eficiente de dos etapas para la síntesis de 7-cloro-4-oxo-1,4-dihidro-1,8-naftiridin-3-carboxilato de etilo. Comparado con la reacción de Grohe-Heitzer reportada, el tiempo y los pasos de reacción se redujeron empleando irradiación de microondas. Este compuesto es un importante intermediario para la preparación de derivados de naftiridonas, los cuáles han recibido una considerable atención debido a su amplio espectro de actividad biológica. Palabras clave: Reacción de Grohe-Heitzer; 1,8-naftiridona; irradiación de microondas; equilibrio tautomérico.<smiles>[R]c1nc2c(cc1F)c(=O)c(C(=O)O)cn2[R2]</smiles>

Nalidixic acid

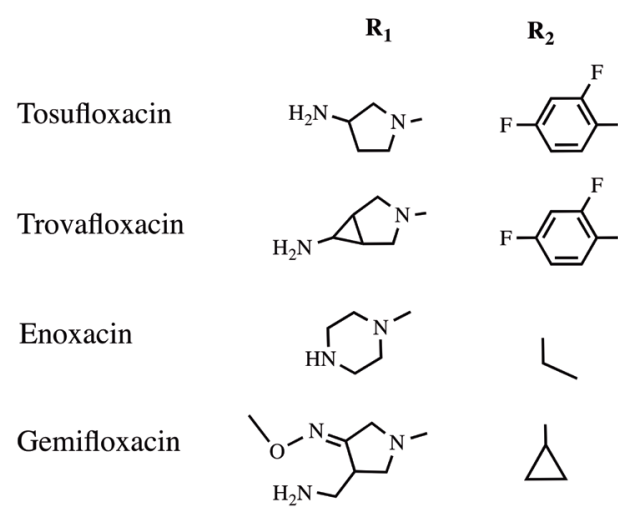

Figure 1. Some antibacterial naphthyridones.

7-chloro-4-oxo-1,4-dihydro-1,8-naphthyridine-3-carboxylate reported in the literature $[5,6,20,21]$.

\section{Results and Discussion}

The 1,8-naphthyridine ring can be prepared by two-step synthesis of $\mathbf{3}$ as showed on Scheme 1. First, 2,6-dichloronicotinic 
acid (1) is reacted with 1,1-carbonyldiimidazol (CDI) to give an imidazolide intermediate at room temperature and 2-2.5 h, according to the literature $[5,6,21]$. Which reacts with ethyl malonate potassium, $\mathrm{MgCl}_{2}$ and triethylamine to generate ethyl 3-(2,6-dichloropyridin-3-yl)-3-oxopropanoate (2) using intervals of room temperature to $60^{\circ} \mathrm{C}$ and $1-15 \mathrm{~h}$ as reactions conditions, reaching $59-93 \%$ yield. Nevertheless, these conditions were improved using microwave radiation, decreasing the reaction time to $5 \mathrm{~min}$ for the synthesis of imidazolide intermediate, the rest of the reaction was performed according to a previous report described in the literature with a $93 \%$ yield.

Then, a usual route of synthesis proposes that molecule 2 reacts with acetic anhydride and triethyl orthoformate, to generate an intermediate vinyl ether in 55 to $90 \%$ yield, using a nitrogen atmosphere and temperatures between $130-140{ }^{\circ} \mathrm{C}$. Following by the addition of an amine or amine substituted at room temperature for $1-5 \mathrm{~h}$ or overnight, resulting 55 to $90 \%$ yield. After this, the synthesized intermediate proceeds to cyclization reaction using $50-60^{\circ} \mathrm{C}$ and time lapse of 1-3 h, with $63-92 \%$ yield of compound 3 . A pathway with three-step synthesis that can be easily converted into one-pot synthesis. The molecule 2 reacts with acetic anhydride and triethyl orthoformate under $\mathrm{MW}$ conditions $\left(70 \mathrm{~W}, 140{ }^{\circ} \mathrm{C}, 3.6 \mathrm{~min}\right.$ ) to yield an intermediate vinyl ether in which $\mathrm{CeCl}_{3}$ and either $\mathrm{NH}_{3}$ or $\left(\mathrm{NH}_{4}\right)_{2} \mathrm{CO}_{3}$ was added to produce a vinyl amine using $100 \mathrm{~W}$ and $100{ }^{\circ} \mathrm{C}$ that allowed easily undergoes cyclization at the same time. The crude product obtained, was purified by a simple column chromatography using hexane to obtain the 1,8-naphythyridone product 3 . The yields using $\mathrm{NH}_{3}$ or $\left(\mathrm{NH}_{4}\right)_{2} \mathrm{CO}_{3}$ were $73 \%$ and $75 \%$ respectively.

Compounds $\mathbf{2}$ and $\mathbf{3}$ were confirmed by ${ }^{1} \mathrm{H}$ NMR and FTIR spectroscopy [19], both presented a keto-enol tautomeric equilibrium as shown in Scheme 2. Tautomer 2a had a $\mathrm{CH}_{2}$ signal (4.09 ppm) while 2b gave a broad $\mathrm{OH}$ signal (12.54 ppm) and a vinyl $\mathrm{H}$ signal (5.72 ppm). In the case of $\mathbf{3 a}$, a vinyl $\mathrm{H}$ signal $(8.53 \mathrm{ppm})$ and a broad $\mathrm{NH}$ signal $(1.59 \mathrm{ppm})$ were observed while $3 \mathbf{b}$, an $\mathrm{OH}$ signal (2.57 ppm) was obtained. According to an extensive aromatic system and intramolecular hydrogen bond, the enol tautomer $\mathbf{3 b}$ was strongly favored. Furthermore, the FT-IR spectra of $\mathbf{2}$ and $\mathbf{3}$ showed absorbance bands corresponding to the presence of $\mathrm{O}-\mathrm{H}$ alcohol, $\mathrm{C}=\mathrm{O}$ enolic ester, as well as a $\mathrm{C}=\mathrm{O}$ ester and a ketone.

\section{Experimental}

The reactions were carried out using a CEM monomode MW synthesizer with a CEM Discover System workstation and monitored by thin-layer chromatography (TLC) using silica gel 60 F254 plates, eluting with hexane/ethylacetate. FT-IR spectra were obtained in $\mathrm{KBr}$ pellets on a Fourier Nicolet (iS10 FT-IR) spectrometer. NMR spectra were recorded on a Varian Mercury Plus $400 \mathrm{MHz}$ spectrometer. Mass spectra were done on a JEOL MStation JMS-700 apparatus.

\section{Procedure and spectral data}

\section{Ethyl 3-(2,6-dichloropyridin-3-yl)-3-oxopropanoate (2a) and ethyl 3-(2,6-dichloropyridin-3-yl)-3-hydroxyacrylate (2b)}

To a solution of 2,6-dichloronicotinic acid $(0.20 \mathrm{~g}, 1.04 \mathrm{mmol})$ in THF- $\mathrm{CH}_{3} \mathrm{CN}(1: 1,3.0 \mathrm{~mL})$ was added 1,1-carbonyldiimidazole $(0.19 \mathrm{~g}, 1.17 \mathrm{mmol})$. The resulting mixture was stirred and heated using MW ( 5 Watts power at $50^{\circ} \mathrm{C}$ for $5 \mathrm{~min}$ ). This crude imidazolide solution was used without purification in the following step. To a solution of ethyl malonate potassium salt (0.19 g, $1.09 \mathrm{mmol})$ in $\mathrm{CH}_{3} \mathrm{CN}(2.50 \mathrm{~mL})$ was added dropwise a mixture of $\mathrm{MgCl}_{2}(0.14 \mathrm{~g}, 1.52 \mathrm{mmol})$ and $\mathrm{Et}_{3} \mathrm{~N}(1.50 \mathrm{~mL})$ under ice cooling. After the mixture was stirred at room temperature for $5 \mathrm{~h}$, the imidazolide was added. The reaction mixture was stirred at room temperature for $15 \mathrm{~h}$, poured onto ice water $(5$ $\mathrm{mL}$ ) resulting in a $\mathrm{pH}$ about 11 , so we acidified this mixture using concentrated $\mathrm{HCl}$ to $\mathrm{pH}=6$. The crude product was extracted with AcOEt, dried with $\mathrm{Na}_{2} \mathrm{SO}_{4}$ and concentrated under vacuum to give product 2, obtained as a yellow oil ( $93 \%$ yield). The product consisted of a mixture of the ketone $2 \mathrm{a}$ and the enol 2b tautomers (confirmed by spectroscopic methods) [6].

IR (KBr) $\mathrm{n}_{\max } 3133$ (C-H aromatic), 2984, 2936 (C-H aliphatic), 1761 ( $\mathrm{C}=\mathrm{O}$ ester $), 1736(\mathrm{C}=\mathrm{O}$ ketone $), 1631(\mathrm{C}=\mathrm{O}$ ester enolic), $1573(\mathrm{C}=\mathrm{C}$ aromatic), $1541(\mathrm{C}=\mathrm{N}$ aromatic $), 1385$ and 1341 (C-N aromatic), 1282, 1242 and 1141 (C-O ester and alcohol), 1095 and 1020 (C-Cl aromatic), 843 and $772(\mathrm{C}-\mathrm{H}$<smiles>O=C(O)c1ccc(Cl)nc1Cl</smiles>

1

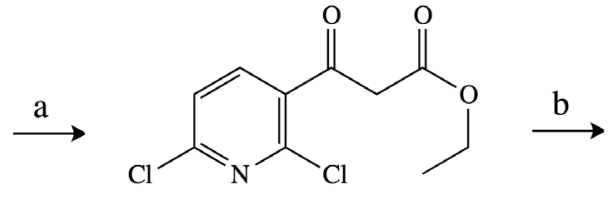

2<smiles>CCOC(=O)c1c[nH]c2nc(Cl)ccc2c1=O</smiles>

3

(a) 1) $\mathrm{CDI}, \mathrm{THF} / \mathrm{CH}_{3} \mathrm{CN}, \mathrm{MW}, 5 \mathrm{~min}$; 2) $\mathrm{EtOCOCH}_{2} \mathrm{COOK}, \mathrm{MgCl}_{2}, \mathrm{Et}_{3} \mathrm{~N}$, RT;

(b) 1) $\left.\mathrm{CH}(\mathrm{OEt})_{3}, \mathrm{Ac}_{2} \mathrm{O}, \mathrm{MW}, 3.6 \mathrm{~min}, 2\right) \mathrm{CeCl}_{3}, \mathrm{NH}_{3}$ or $\left(\mathrm{NH}_{4}\right)_{2} \mathrm{CO}_{3} / 1,4$-dioxane, $\mathrm{MW}, 15$ min.

Scheme 1. Microwave-assisted synthesis of ethyl 7-chloro-4-oxo-1,4-dihydro-1,8-naphthyridine-3-carboxylate. 
Socorro Leyva-Ramos et al.

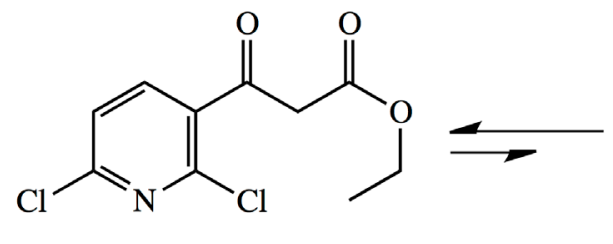

$\mathbf{2 a}$<smiles>CCOC(=O)/C=C(\O)c1ccc(Cl)nc1Cl</smiles>

2b<smiles>CC#CC#CC</smiles>

3a<smiles>CCOC(=O)c1cnc2nc(Cl)ccc2c1O</smiles>

3b

Scheme 2. Tautomeric equilibrium.

aromatic out of plane) $\mathrm{cm}^{-1}$. MS, $m / z(\%): 262.0\left(\mathrm{M}^{+}, 21\right), 216$ (13), 167 (32), 149 (85), 136 (46).

(2a) ${ }^{1} \mathrm{H} \mathrm{NMR}\left(\mathrm{CDCl}_{3} 400 \mathrm{MHz}\right) \delta 1.26(\mathrm{t}, 3 \mathrm{H}, J=7.20$ Hz), 4.09 (s, 2 H), 4.20 (q, $2 \mathrm{H}, J=7.20 \mathrm{~Hz}), 7.40$ (d, $1 \mathrm{H}, J=$ $8.0 \mathrm{~Hz}), 7.98(\mathrm{~d}, 1 \mathrm{H}, J=8.0 \mathrm{~Hz}) .{ }^{13} \mathrm{C} \mathrm{NMR}\left(\mathrm{CDCl}_{3} 400 \mathrm{MHz}\right)$ $\delta 192.41(\mathrm{C}=\mathrm{O}$ ketone $), 172.39(\mathrm{C}=\mathrm{O})$ ester, 152.93, 141.69, 141.41, 140.85 and 123.04, (aromatic C), 60.38, $48.68\left(\mathrm{CH}_{2}\right)$ and $14.01\left(\mathrm{CH}_{3}\right)$.

(2b) ${ }^{1} \mathrm{H}$ NMR $\left(\mathrm{CDCl}_{3} 400 \mathrm{MHz}\right) \delta 1.35$ (t, $3 \mathrm{H}, J=7.20$ $\mathrm{Hz}), 4.29$ (q, $2 \mathrm{H}, J=7.20 \mathrm{~Hz}), 5.72$ (s, $1 \mathrm{H}), 7.36$ (d, $1 \mathrm{H}, J=$ $8.0 \mathrm{~Hz}), 7.94(\mathrm{~d}, 1 \mathrm{H}, J=8.0 \mathrm{~Hz}), 12.54(\mathrm{~s}, 1 \mathrm{H}) .{ }^{13} \mathrm{C} \mathrm{NMR}$ $\left(\mathrm{CDCl}_{3} 400 \mathrm{MHz}\right) \delta 171.16(\mathrm{C}=\mathrm{O})$ ester, 166.57 (C-OH alcohol), 152.94, 141.69, 141.41, 132.47 and 123.04 (aromatic C), 123.46 (vinyl $\mathrm{C}$ ), $61.78\left(\mathrm{CH}_{2}\right)$ and $14.16\left(\mathrm{CH}_{3}\right)$.

\section{Ethyl 7-chloro-4-oxo-1,4-dihydro-1,8-naphthyridine-3- carboxylate (3a) and ethyl 7-chloro-4-hydroxy-1,8- naphthyridine-3-carboxylate (3b)}

A mixture of $2(0.40 \mathrm{~g}, 1.50 \mathrm{mmol})$ and acetic anhydride $(0.36 \mathrm{ml}, 2.25 \mathrm{mmol})$ was heated using MW at 70 Watts until it reached $140^{\circ} \mathrm{C}$. Then triethyl ortoformate was added $(0.38 \mathrm{~mL}, 3.75 \mathrm{mmol})$ stirring under MW heating $(70 \mathrm{~W}$, $140^{\circ} \mathrm{C}$ ) for $3.60 \mathrm{~min}$. The reaction mixture changed from yellow to an intense red color. AcOEt and $\mathrm{CH}_{3} \mathrm{CO}_{2} \mathrm{H}$ were distilled off under reduced pressure. The resulting crude product was mixed with $\mathrm{CeCl}_{3} .7 \mathrm{H}_{2} \mathrm{O}(5.60 \mathrm{mg})$ and stirred for $10 \mathrm{~min}$. Then, a solution of either $\mathrm{NH}_{3}$ or $\left(\mathrm{NH}_{4}\right)_{2} \mathrm{CO}_{3}(2.50 \mathrm{mmol})$ in $5.10 \mathrm{~mL}$ of 1,4-dioxane was added in three fractions to the mixture under open vessel MW at 100 Watts until it reached $100^{\circ} \mathrm{C}$ for $15 \mathrm{~min}$. The crude product was purified by column chromatography using hexane as the mobile phase to obtain $\mathbf{3}$ as colorless solid in a mixture of ketone $\mathbf{3 a}$ and enol $\mathbf{3 b}$ tautomers with m.p. $283-286^{\circ} \mathrm{C}$ [20]. $73 \%$ yield was achieved by using $\mathrm{NH}_{3}$, while in the case of $\left(\mathrm{NH}_{4}\right)_{2} \mathrm{CO}_{3}$ the yield was $75 \%$. IR (KBr) $\mathrm{n}_{\max } 3423$ (O-H alcohol), 3071 (C-H aromatic), 2986, 2926 and 2852 (C-H aliphatic), 1723 (C=O ester), 1664 $(\mathrm{C}=\mathrm{O}$ ketone $), 1623(\mathrm{C}=\mathrm{O}$ ester enolic $), 1594(\mathrm{C}=\mathrm{C}$ aromatic), $1550(\mathrm{C}=\mathrm{N}$ aromatic), 1406 (C-N amine), 1385 (C-N aromatic), 1289, 1219 and 1130 (C-O ester and alcohol), 1073 and1016 (C-Cl aromatic), 796 (C-H aromatic out of plane) $\mathrm{cm}^{-1}$. MS, $m / z(\%): 222\left[\left(\mathrm{M}-\mathrm{CO}-\mathrm{H}_{2}\right)^{+}, 9\right], 220$ (16), 167 (20), 154 (100), 149 (79), 137 (55).

(3a) ${ }^{1} \mathrm{H}$ NMR $\left(\mathrm{CD}_{3} \mathrm{COCD}_{3} 400 \mathrm{MHz}\right) \delta 1.41(3 \mathrm{H}, \mathrm{t}, J=9$ $\mathrm{Hz}), 1.59$ (1 H, bs, NH), $4.42(2 \mathrm{H}, \mathrm{q}, J=9 \mathrm{~Hz}), 7.36(1 \mathrm{H}, \mathrm{d}, J$ $=10 \mathrm{~Hz}), 8.15(1 \mathrm{H}, \mathrm{d}, J=10 \mathrm{~Hz}), 8.53(1 \mathrm{H}, \mathrm{s}) .{ }^{13} \mathrm{C} \mathrm{NMR}$ $\left(\mathrm{CDCl}_{3} 400 \mathrm{MHz}\right) \delta 173.86(\mathrm{C}=\mathrm{O}$ ketone $), 164.03(\mathrm{C}=\mathrm{O}$ ester $)$, $159.53,155.80,141.96,123.42$ and 120.56 (aromatic $\mathrm{C}$ ), 142.23 and 116.80 (vinyl C), $62.32\left(\mathrm{CH}_{2}\right)$ and $14.10\left(\mathrm{CH}_{3}\right)$.

(3b) ${ }^{1} \mathrm{H}$ NMR $\left(\mathrm{CD}_{3} \mathrm{COCD}_{3} 400 \mathrm{MHz}\right) \delta 1.40(3 \mathrm{H}, \mathrm{t}, J=9$ $\mathrm{Hz}), 2.57$ (1 H, s, OH), $4.42(2 \mathrm{H}, \mathrm{q}, J=9 \mathrm{~Hz}), 7.27(1 \mathrm{H}, \mathrm{s})$, $7.46(1 \mathrm{H}, \mathrm{d}, J=10 \mathrm{~Hz}), 8.52(1 \mathrm{H}, \mathrm{d}, J=10 \mathrm{~Hz}) .{ }^{13} \mathrm{C} \mathrm{NMR}$ $\left(\mathrm{CDCl}_{3} 400 \mathrm{MHz}\right) \delta 163.46(\mathrm{C}=\mathrm{O}$ ester $), 163.08(\mathrm{C}-\mathrm{OH}$ alcohol), 156.67, 154.95, 148.29, 139.06, 122.78, 111.17 and 110.92 (aromatic $\mathrm{C}), 62.12\left(\mathrm{CH}_{2}\right)$ and $14.15\left(\mathrm{CH}_{3}\right)$.

\section{Conclusion}

An improved Grohe-Heitzer methodology [21-24] was developed by the use of microwave energy reducing $2.5 \mathrm{~h}$ to 5 min for the synthesis of the intermediate imidazolide. Which was employed in the generation of ethyl 3-(2,6-dichloropyridin-3-yl)-3-oxopropanoate with $93 \%$ yield, while the preparation of ethyl 7-chloro-4-oxo-1,4-dihydro-1,8-naphthyridine-3-carboxylate was made as one-pot reaction with $73 \%$ yield resulting in shorter reaction times and cleaner products. This useful alternative procedure could be helpful to prepare the skeleton of 1,8-naphthyridone agents. 


\section{Acknowledgment}

We wish to thank CONACyT for their financial support (Grant $82585)$ as well as scholarship (218200).

\section{References}

1. Lesher, G. Y.; Froelich, E. J.; Gruett, M. D.; Bailey, J. H.; Brundage, R. P. J. Med. Chem., 1962, 5, 1063-1065.

2. Ahmed, N. S.; Alfooty, K. O.; Khalifah, S.S. J. Chem., 2014, 2014, 1-8.

3. Feng, L. S.; Lv, K.; Liu, M. L.; Wang, S.; Zhao, J.; You, X.; Li, S. J.; Cao, J.; Guo, H. Y. Eur. J. Med. Chem., 2012, 55, 125-136.

4. Lv, K.; Liu, M.; Feng, L.; Sun, L.; Sun, Y.; Wei, Z.; Guo, H. Eur. J. Med. Chem., 2012, 47, 619-625.

5. Tomita, K.; Tsuzuki, Y.; Shibamori, K.; Tashima, M.; Kajikawa, F.; Sato, Y.; Kashimoto, S.; Chiba, K.; Hino, K. J. Med. Chem., 2002, 45, 5564-5575.

6. Tsuzuki, Y.; Tomita, K.; Shibamori, K.; Sato, Y.; Kashimoto, S.; Chiba, K. J. Med. Chem., 2004, 47, 2097-2109.

7. Srivastava, S. K.; Jha, A.; Agarwal, S. K.; Mukherjee, R.; Burman, A. C. Anticancer Agents Med. Chem., 2007, 7, 685-709.

8. Advani, R. H.; Hurwitz, H. I.; Gordon, M. S.; Ebbinghaus, S. W.; Mendelson, D. S.; Wakelee, H. A.; Hoch, U.; Silverman, J. A.; Havrilla, N. A.; Berman, C. J.; Fox, J. A.; Allen, R. S.; Adelman, D. C. Clin. Cancer Res., 2010, 16, 2167-2175.

9. Hwang, Y. J.; Chung, M. L.; Sohn, U. D.; Im, C. Korean J. Physiol. Pharmacol., 2013, 17, 517-523.

10. Massari, S.; Daelemans, D.; Barreca, M.; Knezevich, A.; Sabatini, S.; Cecchetti, V.; Marcello, A.; Pannecouque, C.; Tabarrini, O. J. Med. Chem., 2010, 53, 641-648.

11. Donalisio, M.; Massari, S.; Argenziano, M.; Manfroni, G.; Cagno, V.; Civra, A.; Sabatini, S.; Cecchetti, V.; Loregian, A.; Cavalli, R.; Lembo, D.; Tabarrini, O. J. Med. Chem., 2014, 57, 5649-5663.
12. Dinakaran, M.; Senthikumar, P.; Yogeeswari, P.; Sriram, D. Biomed. Pharmacother., 2009, 63, 11-18.

13. Aboul-Fadl, T.; Bin-Jubair, F. A. S.; Aboul-Wafa, O. Eur. J. Med. Chem., 2010, 45, 4578-4586.

14. Manera, C.; Cascio, M. G.; Benetti, V.; Allará, M.; Tuccinardi, T.; Martinelli, A.; Saccomanni, G.; Vivoli, E.; Ghelardini, C.; Di Marzo, V.; Ferrarini, P. L. Bioorg. Med. Chem. Lett., 2007, 15, 6505-6510.

15. Srivastava, S.K.; Jaggi, M.; Singh, A.T.; Madan, A.; Rani, N.; Vishnoi, M.; Agarwal, S.K.; Mukherjee, R.; Burman, A.C. Bioorg. Med. Chem. Lett., 2007, 17, 6660-6664.

16. Kumar, V.; Jaggi, M.; Singh, A. T.; Maddan, A.; Sanna, V.; Singh, P.; Sharma, P. K.; Irchhaiya, R.; Burman, A. C. Eur. J. Med. Chem., 2009, 44, 3356-3362.

17. Haynes, N. E.; Scott, N. R.; Chen, L. C.; Janson, C. A.; Li, J. K.; Lukacs, C. M.; Raikar, A.; Tozzo, E.; Whittard, T.; Brown, N. F.; Cheung, A. W.; ACS Med. Chem. Lett., 2012, 3, 764-768.

18. Mitscher, L. Chem. Rev., 2005, 105, 559-592.

19. Odagiri, T.; Inagaki, H.; Sugimoto, Y.; Nagamochi, M.; Miyauchi, R.; Kuroyanagi, J.; Kitamura, T.; Komoriya S.; Takahashi, H. J. Med. Chem. 2013, 56, 1974-1983.

20. Hirose, T.; Mishio, S.; J. Matsumoto, J.; Minami, S. Chem. Pharm. Bull. 1982, 30, 2399-2409.

21. Sriram, D.; Senthilkumar, P.; Dinakaran, M.; Yogeeswari, P.; China, A.; and Nagaraja, V. J. Med. Chem., 2007, 50, 6232-6239.

22. Grohe, K.; and Heitzer, H. Liebigs Ann. Chem., 1987, 1987, 29-37.

23. Baker, W.; Cai, S.; Dimitroff, M. Fang, L.; Huh, K.; Ryckman, D.; Shang, X.; Shawar, R.; and Therrien, J. J. Med. Chem., 2004, 47, 4693-4709.

24. Horta, P.; Kuş, N.; Henriques, M. S.; Paixão, J. A.; Coelho, L.; Nogueira, F.; O’Neill, P. M.; Fausto, R.; Santos Cristiano, M. L. J. Org. Chem., 2015, 80, 12244-12257. 